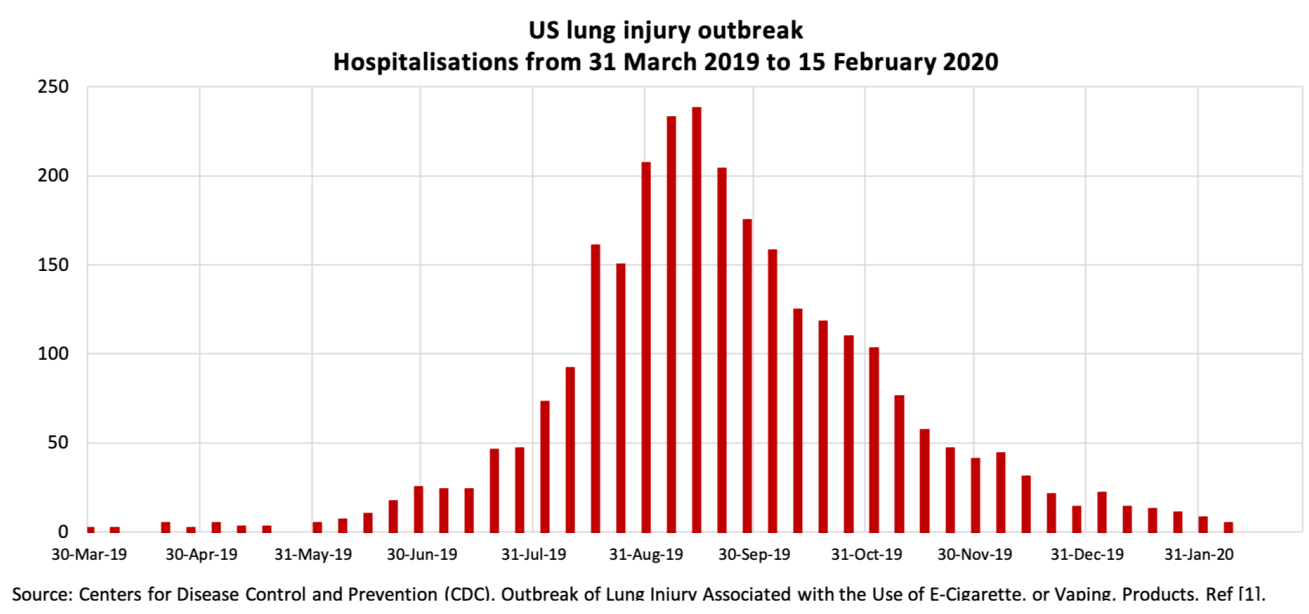

\title{
The outbreak of lung injuries often known as "EVALI" was nothing to do with nicotine vaping
}

\author{
Clive Bates
}

Funding: The author received no specific funding for this work.

Potential competing interests: The author has declared that no potential financial competing interests exist. The author has prior policy positions: the author has been an advocate for tobacco harm reduction as a public health strategy since 1998, and supports the displacement of cigarettes by safer nicotine products, including e-cigarettes. The author has also supported legalisation of cannabis for many years.

\section{Abstract}

Introduction. Many commentators, activists, academics and public health agencies continue to assert or imply that an outbreak of severe and fatal lung injuries that occurred primarily in the United States between mid-2019 and early 2020 may have been caused in part by nicotine vaping. This condition is often known as "EVALI" (E-cigarette, or Vaping, product use-Associated Lung Injury), a term coined by the US Centers For Disease Control and Prevention (CDC). Analysis. An examination of the evidence shows thatEVALI cannot have been caused by nicotine vaping The characteristics of the lung injury outbreak are consistent with localised supply chain contamination. The contaminant has been identified and is known to be Vitamin E Acetate. This had been used as a thickener or cutting agent in illicit Tetrahydrocannabinol (THC) cannabis vape pens. This agent cannot be mixed with nicotine vaping liquids and would serve no useful purpose if it could be. No other cause or causal agent has been identified that would both implicate nicotine liquids and be consistent with the geographical and temporal pattern of the lung injury outbreak. There is a vanishingly small chance that a second independent cause would be found in nicotine liquids, coinciding with the same geography, timing and symptoms as Vitamin E Acetate contamination of THC oils. Further, it is implausible that this hypothetical and currently unidentified cause would disappear in early 2020 without some remedial action in nicotine vaping products. But there has been no identifiable remedial action - nicotine vaping products have not changed, but 
EVALI has largely gone. Residual uncertainties are likely to be due to the unreliable testimony of users regarding their use of THC.

Implications. The attribution of nicotine vaping as a cause of EVALI should stop. The term EVALI is misleading and should be retired and replaced. Risk communicators should shoulder their responsibility to correct residual false risk perceptions. There should be an objective inquiry into how EVALI was handled. Serious consideration should be given to the advantages of legalising and regulating cannabis products.

\section{Introduction}

The outbreak of lung injuries in the United States caused over 2,800 hospitalisations and the deaths of 68 mostly young people in the period between March 2019 and February 2020 ( $\underline{\text { CDC }}$. ${ }^{[1]}$ The condition was initially given various labels (VAPI, VALI, VIPI), but CDC settled on E-cigarette, or Vaping, product use-Associated Lung Injury(EVALI) in guidance issued in October 2019. ${ }^{[2]}$ The outbreak generated a large volume of commentary and news coverage about vaping, much of it confusing or misleading (Gartner et al. 2020) ${ }^{[3]}$ and prompted political and regulatory overreactions (Hall et al. 2020). ${ }^{[4]}$ The responses of public health agencies and the media led to adverse changes in the perceptions of nicotine vaping risk in the United States (Dave et al. 2020). ${ }^{[5]}$ There is evidence that EVALI-related communications questioning the safety of e-cigarettes led to an increase in combustible cigarette use (Katchmar et al. 2021). ${ }^{[6]}$ Though the outbreak was almost entirely US-based, it affected risk perceptions beyond the US. For example, risk perceptions were adversely affected in the UK (Tattan-Birch et al. 2020 $)^{[7]}$, where the impact was picked up in annual ASH(UK) YouGov surveys as a negative step-change between 2019 and 2020 in smokers' perceptions of the harms of e-cigarettes (see chart below). ${ }^{[8]}$

Figure 9: Smokers' perception of harm from e-cigarettes, Great Britain (2013-2021)

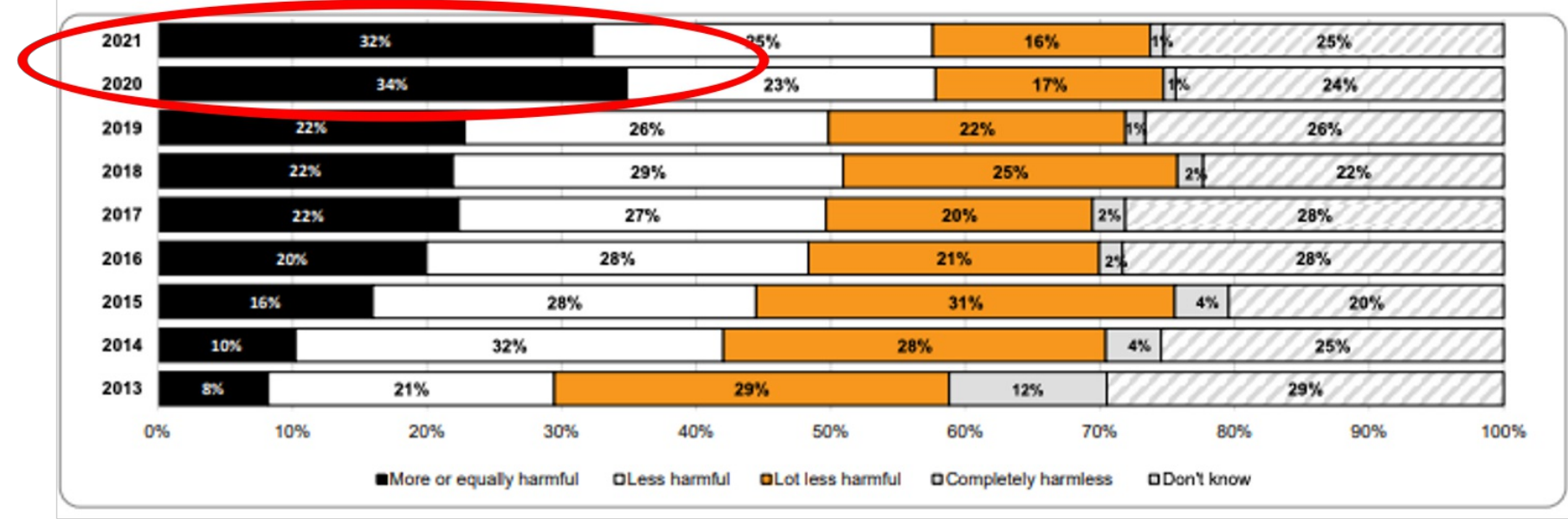

Smokers' changing perception of harm from e-cigarettes 2013-2021: Action on Smoking and Health (UK) / YouGov: reference ${ }^{[8]}$. Author's emphasis in red.

In May 2021, the World Health Organisation's advisory group on the scientific basis for tobacco regulation issued a report with a chapter devoted to EVALI (Kheradmand \& Crotty Alexander). ${ }^{\left[{ }^{[9]}\right.}$ It asserted: It is estimated that 35-40 million adults 
and children globally vape, indicating a large number of people who are vulnerable to EVALI and other vaping-associated health outcomes. [...] The outbreak of EVALI in the USA highlights the importance of broadening the definition of ecigarette toxicity beyond that of smoking, as vaping results in disease risks that are different from those associated with smoking. This advisory group and report are supposed to provide authoritative advice on the scientific basis for tobacco regulation. There is, however, no basis for these statements or several others about EVALI and nicotine vaping in this report. EVALI cannot have been caused by nicotine vaping.

\section{Analysis: why nicotine vaping is not implicated in EVALI}

1. The lung disease outbreak was limited in time from mid-2019 to early-2020, peaking in September 2019 and with cases tailing off in early 2020, to the point that CDC stopped reporting on cases in February 2020 (CDC hospitalisation data - see the cover image for this paper) ${ }^{[1]}$. The EVALI outbreak is essentially over. It did not arise from THC or nicotine vaping products at scale before this period and largely disappeared after this period, though it is possible a few residual cases in 2020 were initially misdiagnosed as COVID-19 (CDC, 2020). ${ }^{[10]}$ The outbreak was also limited in geography, primarily to North America. ${ }^{[11]}$ This geographic and temporal pattern is consistent with a cause related to localised contaminated supply (comparable to a food poisoning outbreak). It means EVALI cannot be intrinsic to nicotine or THC vaping products because it would have been seen at other times and in other places, given nicotine vaping has been a worldwide phenomenon since around 2010. The US lung injury outbreak must have been caused by something about the products available in North America in the latter half of 2019.

2. The contaminating agent has been identified. It is Vitamin E Acetate (VEA) Blount et al. 2020), ${ }^{[12][13]}$ which is used as a thickener or diluent in Tetrahydrocannabinol (THC) cannabis oils. Its exact biochemical interaction with lung tissue is uncertain and is a subject of ongoing work. ${ }^{[14][15]}$ However, there is no real doubt that it played a causal role in EVALI. CDC has acknowledged this: according to Dr Anne Schuchat, CDC's principal deputy director in 2019: "we can conclude that what I call the explosive outbreak of cases of EVALI can be attributed to exposure to THC-containing vaping products that also contained Vitamin E acetate" (CDC published transcript, 20 December 2019) ${ }^{[16]}$

3. Vitamin E Acetate was added to illicit THC vapes as a thickener or 'cutting agent' to dilute the strength of expensive THC-containing oils while maintaining the viscosity of the liquid. THC users regard liquid viscosity as a mark of product potency and quality. The addition of VEA to THC vaping oils served an economic purpose, albeit a criminal and disreputable economic purpose. It is also possible that thickeners with similar chemical or physical properties to VEA could have a role, but none has been definitively identified so far, and attention has focussed exclusively on VEA. The supply chain involves makers of thickeners and final sellers of illicit THC vape pens. The first signs that thickeners were culpable emerged in August 2019. More recently, there have been emerging concerns about another cutting agent using in cannabis liquids, Phytol. ${ }^{[17][18][19]}$ This suggests continued vigilance is required in the illicit THC vaping market. More information on the companies and products involved is available in the specialist cannabis publication, Leafly. [20][21]

4. This agent, Vitamin E Acetate, cannot be added to nicotine e-liquids (Kozlovich et al, 2021). ${ }^{[22]}$ It is not soluble in the 
base liquids, propylene glycol and vegetable glycerin, used for nicotine vaping, and these liquids and VEA would separate if mixed. Further, VEA would serve no useful economic purpose even if it could be added. That is because the nicotine vaping base liquids are cheap and readily available, and it is simple to dilute any nicotine liquid to a desired strength. Also, there is no reason to add any thickener to a nicotine liquid product because liquid viscosity has no significance for nicotine vapers. For nicotine liquids to be implicated in EVALI, a different cause or causal agent would be required. None has been identified. (See further discussion at point 9 below).

5. CDC data show that the EVALI cases had largely cleared up by early 2020 (see title chart above) ${ }^{[1]}$ This is consistent with the North American illicit supply chain steadily emptying of VEA-contaminated THC products. It would be consistent with remedial action taken to address the risk in VEA-contaminated THC vapes by removing the VEA and using different thickeners or no thickeners. The criminal suppliers have strong incentives not to harm or kill their customers, not to increase their criminal liability, and not to degrade consumer confidence in their products. They will have removed VEA as soon as it became clear this was a causal agent in EVALI, probably starting in August 2019. Arrests of illicit operators and closure of illicit suppliers will also have contributed to the decline, and these intensified from September 2019. [23]

6. No equivalent remedial action has been taken with nicotine vaping products. It has not been possible to identify any changes to e-liquid ingredients, devices or manufacturing processes for nicotine vaping products that would have ended their hypothetical role in causing EVALI. Such changes would need to address a speculative cause that remains unknown but is specific to the geography and timing of EVALI. Yet, without any apparent change to nicotine vaping products, EVALI has declined to negligible levels. The absence of any remedial actions reinforces the case that nicotine vaping products never had a role in EVALI.

7. To recap: for nicotine vaping to be implicated in EVALI, there would need to be a cause other than VEA, yet none has so far been identified. That unknown cause would have to have emerged at the same time, in the same limited geography, and cause the same symptoms as VEA exposure. The unidentified cause would have to have been somehow eliminated for the EVALI incidence to decline to trivial levels by February 2020. However, no known remedial action has been taken with nicotine vapes. There is no real chance that any of this happened and no evidence to suggest that it did. It is, therefore, beyond reasonable doubt that nicotine vaping is not implicated in EVALI.

8. There is some uncertainty about attributing all the EVALI cases definitively to contaminated THC vapes. However, that does not justify extending the uncertainty to nicotine vaping for the reasons described in 1-7 above. The major sources of uncertainty are in the sporadic and ad hoc testing of EVALI patients for THC or VEA exposure and the poor reliability of users' accounts of their exposure. It is not surprising that some illicit THC users would deny illicit THC use - it could cause them difficulties (real or perceived) with their parents, school, college, employer, parole officer, or law enforcement. Some EVALI patients have initially denied using THC but changed their account in a more detailed follow-up interview (CDC, 2020) ${ }^{[24]}$ Discordant reporting of cannabis use is common and well-documented Palamar $\underline{\text { \& Le, 2021 }})^{[25]}$ and should be recognised as a limitation by investigators seeking causes of EVALI. This issue was highlighted by Dr. Scott Aberegg, a critical care pulmonologist at University of Utah Health. In a wry comment to CNBC, he stated: "It may turn out there are only two kinds of people who get this disease: those who vape THC and those who won't admit it'. [26] The epidemiological approach described above is a more reliable way to assess the 
causes of the lung injury outbreak than user testimony.

9. Several theories have been advanced for how nicotine vapes could cause acute lung injury. With reference to Maddison et al. 2019, ${ }^{[27]}$ Eissenberg \& Maziak, 2020 ${ }^{[28]}$ suggest exposure to lipids in e-liquids may be a cause, though this is disputed. ${ }^{[29]}$ Hayeck et al. 2020 ${ }^{[30]}$ suggest a possible interaction between e-liquid solvents (PG and VG) and lung tissue surfactants. Kleinman et al. 2020 ${ }^{[31]}$ suggested nickel alloys as a possible cause. This is not an exhaustive review, but these theories and the supporting early-stage research do not establish nicotine vaping as a cause of EVALI, and these results are subject to contested interpretations. More importantly, whatever their merits, these theories cannot explain the following: (1) the geographically localised and time-concentrated nature of the EVALI outbreak; (2) why this would coincide exactly with a THC supply chain problem and cause identical symptoms; or (3) how EVALI cleared up without remedial action to address these possible causes in nicotine vaping. This is not to suggest there is no value in the research cited above; it just cannot explain the pattern of EVALI lung injuries seen in the United States in 2019. Unless carefully reported, such research may lead to doubt where no doubt is justified.

10. There may have been a few cases outside the United States. WHO's advisory committee pointed to isolated cases in Canada, Japan, Mexico and the United Kingdom, though some of these reports predate the description of EVALI. ${ }^{[9]}$ There does not appear to have been a noticeable outbreak outside North America. Isolated cases may arise from international travel or internet commerce in THC vapes. There may be occasional cases of acute respiratory distress syndrome, pneumonia or other severe lung conditions that happen to people who use e-cigarettes but without e-cigarettes being the cause. It is also possible that e-cigarette use might trigger a severe adverse reaction in someone with an underlying vulnerability, allergy, or pre-existing condition. Attribution is challenging and often disputed (Campagna et al. 2016). ${ }^{[32]}$ But such causes would be manifest in a scattering of cases that would not have the particular geographic or temporal concentration associated with localised supply chain contamination that characterises the US EVALI outbreak. There is so far no compelling evidence that such cases are a material problem with nicotine vaping. If there were such evidence, it would be nothing to do with the EVALI outbreak. EVALI, as discussed above, can be explained convincingly and completely as a problem arising from sales of illicit THC vaping products containing oils that had been cut with Vitamin E Acetate. EVALI cannot be explained by the typical components, ingredients and production processes of nicotine vaping products.

\section{Implications}

\section{- Commentators should stop claiming that nicotine vaping is implicated in "EVALI". Agencies, academics,} journals, peer reviewers, health organisations, activist groups, foundations, media commentators, and politicians should stop falsely asserting or implying that nicotine vaping was a cause of EVALI. It is ethically inappropriate to use false or tenuous claims or exaggerated doubt about risks to try to change the behaviour of others. That ethical problem is compounded in this case because of the risk that users would be deterred from switching from high-risk to low-risk nicotine products and would therefore suffer harm based on misleading public health information.

- "EVALI" should be renamed to reflect the real risks. The contrived naming of this condition as "E-cigarette, or 
Vaping, product use-Associated Lung Injury" (EVALI) is misleading, and the language is unnecessarily vague. Users and suppliers of THC vapes do not use the term "e-cigarettes" to describe THC vaping products such as vape pens. The term "e-cigarette" is primarily used to refer to nicotine vaping products that are replacements for cigarettes. ${ }^{[33]}$ It follows that the term EVALI, by implicating e-cigarettes, is misleading to nicotine users. At the same time, THC users are misled by an overly broad definition that seems to include all possible vaping products. But it is clear that the 2019 lung injury outbreak was attributable to a specific cause: Vitamin E Acetate added to THC vaping oils. A researcher who has called for renaming EVALI points out that we would not describe the opioid epidemic as an analgesic epidemic (Foulds, 2020). ${ }^{\left[{ }^{[3]}\right]}$ A new effort should be made to define the best terminology to accurately communicate the nature of the risk to the right target audiences. Given that it coined the phrase EVALI, CDC should take responsibility for changing what has become unambiguously misleading terminology.

- Risk communicators should address the residual false risk perceptions The false risk perceptions created during the EVALI crisis period in 2019 persist today. Yet the causes are now clear and the episode is, for all practical purposes, over and unlikely to recur in the same form. There needs to be a concerted effort to put the record straight and to stop unfounded doubt that is negatively affecting risk perceptions and health behaviours. Journals and authors should review academic papers that refer to EVALI and correct or qualify false or misleading statements. Health organisations, including the World Health Organisation, should review their public health communications and advice to practitioners. Advocacy groups and foundations should correct campaign literature and media or political communications that suggest EVALI was caused by nicotine vaping.

- There should be an inquiry into how EVALI was handled It is not the purpose of this paper to assess or criticise the actions or intentions of anyone involved in the response to EVALI. However, it is important that lessons are learned and there is some accountability in public health. The handling of EVALI may go down as a public health failure, in which nicotine vapers were needlessly frightened away from a beneficial behaviour change, while THC vapers were given inadequately precise risk information. ${ }^{[35]}$ Michael Siegel of the Department of Community Health Sciences, Boston University School of Public Health, provided a high quality and, at times, disturbing real-time commentary on the conduct of experts and agencies in responding to EVALI from August 2019 to January 2020.[36] The specialist Vaping 360 website has chronicled developments over this period and criticised the role played by CDC. ${ }^{[37][38]}$ A more formal investigation, taking evidence from those directly involved, would be a fair and constructive way to identify and learn lessons from the handling of EVALI.

- EVALI strengthens the case for the legalisation and regulation of cannabis products The lung injury outbreak was caused by the reckless profit-seeking behaviour of actors in an illicit supply chain meeting ongoing demand for cannabis from millions of American consumers. Past 30-day cannabis prevalence among US 12th grade students (age 17-18) has been around 20 percent for 25 years, ${ }^{[39]}$ with 11.8 million young adults reporting cannabis use in the past year (2018 data). ${ }^{[40]}$ This high prevalence of use is despite the cannabis trade being illegal at the United States federal level and illegal at the individual state level for most or all of that period. ${ }^{[41]}$ Prohibition does not prevent access to these products, it changes how such products are made available and who supplies them. Smith \& Goniewicz $\underline{2021},{ }^{[42]}$ showed that odds of suffering EVALI were three times higher among users living in states with no legal access to cannabis compared to users in states with active recreational policies. Compared to prohibition, the EVALI 
outbreak suggests it would be better for public health to have a regulatory regime that permitted cannabis products to be made available with acceptable safety standards while minimising risks from rogue producers. Such a regime could require, inter alia, the listing of ingredients, black-listing of hazardous additives, reporting and verification of psychoactive ingredients at known and predictable potency, appropriate warnings, and restrictions on retail availability, marketing and branding. European Union regulation prohibits the use of vitamins in nicotine vaping products ${ }^{[43]}$, though primarily to limit appeal rather than for safety reasons. This may have given some reassurance to nicotine vapers in the EU accessing legal nicotine products. ${ }^{[44][45]}$ However, it would not stop rogue THC vape products from being sold illicitly and without any regulatory supervision. Cannabis legalisation is a multi-faceted issue and cannot be justified or rejected just on the basis of the experience with EVALI alone. However, the experience of EVALI provides an additional consumer protection argument in favour of the legalisation and regulation of cannabis products.

\section{Feedback and review}

The author would welcome feedback and critical and constructive review of this analysis and will update it accordingly.

\section{References}

1. a, b, c U.S. Centers For Disease Control and Prevention (CDC). (2020). Outbreak of Lung Injury Associated with the Use of E-Cigarette, or Vaping, Products.

2. `David A. Siegel, Tara C. Jatlaoui, Emily H. Koumans, Emily A. Kiernan, et al. (2019).Update: Interim Guidance for Health Care Providers Evaluating and Caring for Patients with Suspected E-cigarette, or Vaping, Product Use Associated Lung Injury - United States, October 2019. MMWR Morb. Mortal. Wkly. Rep., vol. 68 (41), $919-927$. doi:10.15585/mmwr.mm6841e3.

3. ^Coral Gartner, Billie Bonevski, Wayne Hall. (2020). Miscommunication about the causes of the US outbreak of lung diseases in vapers by public health authorities and the media. Drug Alcohol Rev., vol. 39 (1), 3-6. doi:10.1111/dar.13024.

4. 'Wayne Hall, Coral Gartner, Billie Bonevski. (2020). Lessons from the public health responses to the US outbreak of vaping-related lung injury. Addiction, vol. 116 (5), 985-993. doi:10.1111/add.15108.

5. `Dhaval Dave, Daniel Dench, Donald Kenkel, Alan Mathios, et al. (2020). News that takes your breath away: risk perceptions during an outbreak of vaping-related lung injuries. J Risk Uncertain, vol. 60 (3), 281-307. doi:10.1007/s11166-020-09329-2.

6. ^Amanda Katchmar, Adrian Gunawan, Michael Siegel. (2021). Effect of Massachusetts House Bill No. 4196 on electronic cigarette use: a mixed-methods study. Harm Reduct J, vol. 18 (1). doi:10.1186/s12954-021-00498-0.

7. ^Harry Tattan-Birch, Jamie Brown, Lion Shahab, Sarah E Jackson. (2020). Association of the US Outbreak of Vaping- 
Associated Lung Injury With Perceived Harm of e-Cigarettes Compared With Cigarettes. JAMA Netw Open, vol. 3 (6), e206981. doi:10.1001/jamanetworkopen.2020.6981.

8. ${ }^{a, b}$ Action on Smoking and Health (UK) \& YouGov. (2021). Use of e-cigarettes among adults in Great Britain, 2021.

9. ${ }^{a}, \mathrm{~b}$ Farrah Kheradmand, Laura E. Crotty Alexander. (4 May 2021).EVALI: e-cigarette or vaping product use-associated lung injury (chapter 12). WHO study group on tobacco product regulation: Report on the scientific basis of tobacco product regulation: eighth report of a WHO study group.

10. 'Christina Armatas, Amy Heinzerling, Jason A. Wilken. (2020). Notes from the Field: E-cigarette, or Vaping. Product Use-Associated Lung Injury Cases During the COVID-19 Response - California, 2020. MMWR Morb. Mortal. Wkly. Rep., vol. 69 (25), 801-802. doi:10.15585/mmwr.mm6925a5.

11. 'Kate Kelland. (14 October 2019). Vaping illness, deaths likely very rare beyond U.S., experts say. Reuters.

12. ^Benjamin C. Blount, Mateusz P. Karwowski, Peter G. Shields, Maria Morel-Espinosa, et al. (2020).Vitamin E Acetate in Bronchoalveolar-Lavage Fluid Associated with EVALI. N Engl J Med, vol. 382 (8), 697-705. doi:10.1056/nejmoa1916433.

13. ^National Center for Biotechnology Information. (Retrieved 23 July 2021).PubChem Compound Summary for CID 86472, alpha-Tocopherol acetate (Vitamin E Acetate).

14. 'Mitchell DiPasquale, Omotayo Gbadamosi, Michael H. L. Nguyen, Stuart R. Castillo, et al. (2020).A Mechanical Mechanism for Vitamin E Acetate in E-cigarette/Vaping-Associated Lung Injury. Chem. Res. Toxicol., vol. 33 (9), 24322440. doi:10.1021/acs.chemrestox.0c00212.

15. ' Dan Wu, Donal F. O'Shea. (2020). Potential for release of pulmonary toxic ketene from vaping pyrolysis of vitamin E acetate. Proc Natl Acad Sci USA, vol. 117 (12), 6349-6355. doi:10.1073/pnas.1920925117.

16. ' U.S. Centers for Disease Control and Prevention. (20 December 2019). Transcript of December 20, 2019. Telebriefing: Update on Lung Injury Associated with E-cigarette Use, or Vaping.

17. 'David Downs. (19 July 2021). What is phytol and is it safe to vape?. Leafly.

18. 'Daniela Schwotzer, Andrew Gigliotti, Hammad Irshad, Wendy Dye, et al. (2021).Phytol, not propylene glycol, causes severe pulmonary injury after inhalation dosing in Sprague-Dawley rats. Inhalation Toxicology, vol. 33 (1), 33-40. doi:10.1080/08958378.2020.1867260.

19. ^National Center for Biotechnology Information. (Retrieved 23 July 2021).PubChem Compound Summary for CID 5280435, Phytol.

20. `David Downs. (30 August 2019). Vape pen lung disease has insiders eyeing misuse of new additives. Leafly.

21. `David Downs. (10 January 2020). Vape pen lung injury: Here's what you need to know. Leafly.

22. 'Shannon Kozlovich,; Arit M Harvanko; Neal L Benowitz. (2021).Vitamin E Acetate is not Soluble in Nicotine E-liquids. Tobacco Regulatory Science, vol. 7(2) pp 130-134 .

23. ^Allison Aubrey. (27 September 2019). Many Vaping Illnesses Linked To Black Market 'Dank Vapes' Or Other THC Products. National Public Radio (US).

24. 'Isaac Ghinai, Livia Navon, Jayleen K.L. Gunn, Lindsey M. Duca, et al. (2020). Characteristics of Persons Who Report Using Only Nicotine-Containing Products Among Interviewed Patients with E-cigarette, or Vaping. Product UseAssociated Lung Injury - Illinois, August-December 2019. MMWR Morb. Mortal. Wkly. Rep., vol. 69 (3), 84-89. 
doi:10.15585/mmwr.mm6903e1.

25. ^Joseph J. Palamar, Austin Le. (2021). Discordant Reporting of Vaping of Cannabis among High School Seniors in the United States. The American Journal of Drug and Alcohol Abuse. doi:10.1080/00952990.2021.1942030.

26. 'Angelo LeVito. (20 November 2019). Doctors treating deadly lung disease face a problem: Some patients lie about vaping. CNBC (news website).

27. 'Matthew C. Madison, Cameron T. Landers, Bon-Hee Gu, Cheng-Yen Chang, et al. (2019).Electronic cigarettes disrupt lung lipid homeostasis and innate immunity independent of nicotine. doi:10.1172/jci128531.

28. 'Thomas Eissenberg, Wasim Maziak. (2020). Are Electronic Cigarette Users at Risk for Lipid-mediated Lung Injury?. Am J Respir Crit Care Med, vol. 201 (8), 1012-1013. doi:10.1164/rccm.201910-2082le.

29. 'Clive Bates, Konstantinos Farsalinos, Riccardo Polosa. (2020). Comments on: Are Electronic Cigarette Users at Risk for Lipid-mediated Lung Injury?. PubPeer.

30. ^Nathalie Hayeck, Carl Zoghzoghi, Ebrahim Karam, Rola Salman, et al. (2021). Carrier Solvents of Electronic Nicotine Delivery Systems Alter Pulmonary Surfactant. Chem. Res. Toxicol., vol. 34 (6), 1572-1577. doi:10.1021/acs.chemrestox.0c00528.

31. 'Michael T. Kleinman, Rebecca Johnson Arechavala, David Herman, Jianru Shi, et al. (2020).E-cigarette or Vaping Product Use-Associated Lung Injury Produced in an Animal Model From Electronic Cigarette Vapor Exposure Without Tetrahydrocannabinol or Vitamin E Oil. JAHA, vol. 9 (18). doi:10.1161/jaha.120.017368.

32. 'Davide Campagna, Maria Domenica Amaradio, Mark F. Sands, Riccardo Polosa. (2016). Respiratory infections and pneumonia: potential benefits of switching from smoking to vaping. pneumonia, vol. 8 (1). doi:10.1186/s41479-0160001-2.

33. ^Robert West. (2019). E-cigarette. doi:10.32388/484818.

34. 'Jonathan Foulds. (7 December 2020). Lets call EVALI what it is: THC VALI (rapid response to BMJ case report: Vaping-induced lung injury in a 21-year-old woman). BMJ.

35. 'Clive Bates. (13 April 2020). US vaping lung injury outbreak was a public health fiasco or worse - comment to FDA. Counterfactual.

36. 'Michael Siegel. (August 2019-January 2020). The Rest of the Story: Tobacco and Alcohol News Analysis and Commentary. Blog.

37. `Jim McDonald. (27 February 2020). Its Reputation Stained, CDC Closes the Books on "EVALI”. Vaping 360.

38. `Jim McDonald. (8 May 2020). A Look Back at CDC's Response to the 2019 "EVALI" Lung Injuries. Vaping 360.

39. ^Richard A. Miech (Principal Investigator). (15 December 2020). Trends in 30-Day Prevalence of Use of Various Drugs in Grades 8, 10, and 12, 1991-2020 (Table 3). Monitoring the Future. University of Michigan.

40. 'U.S. National Institute on Drug Abuse. (July 2020). Marijuana Research Report What is the scope of marijuana use in the United States?

41. 'Wikipedia. (Accessed 26 July 2021). Timeline of cannabis laws in the United States. Wikipedia Free Encyclopaedia.

42. `Danielle M. Smith, Maciej L. Goniewicz. (2020). E-cigarette or vaping product use-associated lung injury and statelevel cannabis policies. J Cannabis Res, vol. 2 (1). doi:10.1186/s42238-020-00053-x.

43. ^European Union. (3 April 2014). Tobacco Products Directive 2014/40/EU Article 7(6)(a). 
44. `John Newton. (29 October 2019). Vaping and lung disease in the US: PHE's advice. Health Matters. Public Health England.

45. ^Blessing Nyakutsikwa, John Britton, Ilze Bogdanovica, Tessa Langley. (2020).Vitamin E acetate is not present in licit e-cigarette products available on the UK market. Addiction, vol. 115 (4), 782-783. doi:10.1111/add.14920. 